\title{
LEVEL STATISTICS INSIDE THE VORTEX OF A SUPERCONDUCTOR AND SYMPLECTIC RANDOM MATRIX THEORY IN AN EXTERNAL SOURCE
}

\author{
E. Brézin ${ }^{1}$, S. Hikami ${ }^{2}$ and A. I. Larkin ${ }^{c), d)}$ \\ ${ }^{1}$ Laboratoire de Physique Théorique, Ecole Normale Supérieure, 24 rue Lhomond 75231, Paris \\ Cedex 05, France* \\ 2 Department of Pure and Applied Sciences, University of Tokyo \\ Meguro-ku, Komaba, Tokyo 153, Japan \\ c) Theoretical Physics Institute, University of Minnesota, Minneapolis, Minnesota 55455, USA \\ d) Landau Institute for Theoretical Physics, 117334 Moscow, Russia
}

\begin{abstract}
In the core of the vortex of a superconductor, energy levels appear inside the gap. We discuss here through a random matrix approach how these levels are broadened by impurities. It is first shown that the level statistics is governed by an ensemble consisting of a symplectic random potential added to a nonrandom matrix. A generalization of previous work on the unitary ensemble in the presence of an external source (which relied on the Itzykson-Zuber integral) is discussed for this symplectic case through the formalism introduced by Harish-Chandra and Duistermaat-Heckman. This leads to explicit formulae for the density of states and for the correlation functions, which describe the cross-over from the clean to the dirty limits.
\end{abstract}

Typeset using REVTEX

*Unité propre du centre national de la Recherche Scientifique, Associée à l'Ecole Normale Supérieure et à l'Université de Paris-Sud 


\section{INTRODUCTION}

The energy levels inside a vortex of a superconductor have been characterized long ago [1], but recent studies have dealt with the broadening of these levels by impurities. The density of states has been computed both in the clean limit and for the dirty case.

In this article, we discuss the crossover between these two limits. The impurity potential is handled through a random matrix theory. However the matrix elements between different energy levels due to the impurities are strongly correlated, and therefore one is far from a usual Wigner-Dyson random matrix theory.

It is shown below that the crossover is characterized in this case by a random symplectic matrix coupled to an external non-random source matrix. For handling this problem we develop a technique which generalizes earlier work on the unitary ensemble in which one considered the cross-over from a deterministic hamiltonian to a fully random hermitian hamiltonian [2 5]. In the hermitian case, the formalism relied on an integral over the unitary group due to Harish-Chandra [6] and rederived in the context of random matrix theory by Itzykson-Zuber [7]. All the correlation functions for the energy levels were then found explicitely. For the vortex problem considered here the perturbation due to impurities is a matrix with a symplectic structure, which we treat as a random potential added to the unperturbed diagonal matrix consisting of the regularly spaced energy levels. It is shown below that for this problem a similar integral over the symplectic group, considered by Harish-Chandra [6], and more recently generalized by Duistermaat and Heckman [8] leads also to explicit formulae. The crossover from the clean spectrum to the dirty limit follows

from this formalism. We will show how this general behavior for the crossover is relevant to the actual problem of the excitation energy spectrum in a superconducting vortex.

\section{SYMPLECTIC STRUCTURE OF THE PERTURBATION FOR THE ENERGY LEVELS INSIDE A VORTEX}

The energy eigenvalues and eigenfunctions of a quasi-particle inside the vortex of a superconductor, were obtained long ago [1]. The two component wave functions of excitations $\hat{\psi}=\left(\psi_{1}, \psi_{2}\right)$ in a superconductor satifies the Bogolubov-de Gennes equation,

$$
\left[\sigma_{z}\left(H_{0}+V_{i m p}\right)+\sigma_{x} \operatorname{Re} \Delta(r)+\sigma_{y} \operatorname{Im} \Delta(r)\right] \hat{\psi}=E \hat{\psi}
$$

where $H_{0}=p^{2} / 2 m-E_{F}$, and $\Delta(r)$ is a gap order parameter. The impurity potential $V_{i m p}$ is a sum of short range scattering sites $r_{i}$ :

$$
V_{i m p}(r)=\sum_{i} V_{i} \delta\left(r-r_{i}\right)
$$

In the absence of impurity, the Schrodinger equation (2.1) leads to a spectrum of equidistant states $E_{n}^{0}$

$$
E_{n}^{0}=-\hbar \omega_{0}\left(n-\frac{1}{2}\right)
$$


where $n$ is an integer defining the quantized angular momentum of the vortex, and $\hbar \omega_{0}=$ $\Delta^{2} / E_{F}$. We assume that the spacing $\hbar \omega_{0}$ between these levels is much smaller than the gap $\Delta$, and consequently there are many excitation levels in the vortex core.

Using the explicit wave functions for the unperturbed eigenstates, one finds that the matrix elements of the interaction due to the $i$-th impurity are

$$
A_{n m}^{i}=\frac{V_{i} e^{-2 K\left(r_{i}\right)}}{\lambda_{F} \xi} e^{i(m-n) \phi_{i}}\left[J_{n}\left(k_{F} r_{i}\right) J_{m}\left(k_{F} r_{i}\right)-J_{n-1}\left(k_{F} r_{i}\right) J_{m-1}\left(k_{F} r_{i}\right)\right]
$$

where $\left(r_{i}, \phi_{i}\right)$ is the position of the i-th impurity in polar coordinates. The indices $n$ and $m$ run from $-N$ to $+N$ where $N \sim \Delta / \omega_{0} \sim E_{F} / \Delta$ is assumed to be a large number.

The scattering time $\tau$, given by $1 / \tau=2 n_{i} V_{i}^{2} m$, where $n_{i}=N_{i} / \xi^{2}$ is the density of impurities in the vortex ; $N_{i}$ the total of impurities in the cross-section of a vortex of radius $\xi$.

Three different situations may occur: a) a dirty limit $\Delta<<1 / \tau<<E_{F}$, b) a clean limit $\omega_{0}<1 / \tau<<\Delta$, c) a superclean case $1 / \tau<<\omega_{0} \sim \Delta^{2} / E_{F}$. However, in [11, [12] it has been shown that the level statistics depends not only on the parameter $\tau$ but also on the number of impurities $N_{i}$. This is clear in the superclean case. Then the density of states has narrow peaks centered around the unperturbed energy levels (2.3). The form of these peaks is Gaussian when the number of impurities is large ; however as shown in [14,12] these peaks have a different form when there is only a single impurity.

In the clean case $\omega_{0}<1 / \tau<\Delta$, the level statistics also depends upon the number of impurity $N_{i}$. If $N_{i} \rightarrow \infty$ and if the random potential is a white noise, it was shown in 13 that one can apply standard random matrix theory. However if the number of impurities $N_{i}$ is smaller than a certain number $N_{i c}\left(N_{i c}>\left(E_{F} / \Delta\right)^{1 / 2}\right)$, Koulakov and Larkin [11 have found that the spectrum is of a different type.

In the present article, we have defined the dirty and the clean limits by $N_{i}>N_{i c}$, and $N_{i}<N_{i c}$ respectively. (These definitions differ slightly from the usual ones, quoted hereabove.)

When there is only one impurity in the vortex core, with a radius $\xi$, the impurity is located at a position $r_{i} \sim \xi$. We are thus in a limit in which $k_{F} r_{i}>>1$, since the correlation length is $\xi \sim \hbar^{2} k_{F} / m \Delta$, and we have thus $k_{F} \xi \sim k_{F}^{2} / \Delta \sim E_{F} / \Delta>>1$. Therefore, in the clean limit, this condition is always satisfied. In the dirty limit, the number of impurities increase and the typical value of the quantity $k_{F} r_{i}$ is reduced.

In that limit the Bessel functions in (2.4) may be replaced by their asymptotic expansions and the matrix element $A_{n m}^{i}$, produced by the scattering site $i$ between two states $n$ and $m$, is given for $k_{F} r_{i} \gg 1$ by

$$
A_{n m}^{i} \simeq \tilde{C} e^{i(m-n) \phi_{i}} \sin \left(2 k_{F} r_{i}-\frac{n+m}{2} \pi\right)
$$

where $\tilde{C}=\frac{V_{i} e^{-2 K\left(r_{i}\right)}}{\lambda_{F} \xi}\left(\frac{2}{\pi k_{F} r_{i}}\right)$.

The corresponding secular equation for the clean case was solved by Koulakov and Larkin [11] who have found that, after averaging over the location of the impurity, the density of states $\rho(E)$ was given by

$$
\rho(E)=\frac{2}{\omega_{0}} \sin ^{2}\left(\frac{\pi E}{\omega_{0}}\right)
$$


(normalized to one after integration on the interval $0<E<\omega_{0}$ ).

In the dirty limit, $k_{F} r_{i}$ is not as large and it is necessary to take into account the subasymptotic behavior of the Bessel functions, and to sum over the impurities. Since the matrix elements $A_{n m}^{i}$ have an oscillatory behavior, it is natural to regard them in the dirty limit as the elements of a random matrix. However it is necessary to keep some of the structure exhibited by the clean limit (2.5) in mind.

In the dirty limit or in the moderate clean case, a random matrix theory has already been considered for the superconductor-normal interface, and for a quantum dot with a superconductor boundary. [15,16]. The excitation levels of the superconductor vortex have also been analysed in this way [13]. The random matrix ensemble, for such cases, has been suggested to be invariant by the symplectic group $S p(N)$.

If the full Hamiltonian is treated as a random matrix in a symplectic ensemble $S p(n)$, which is valid in the extreme dirty limit, the density of states has been conjectured 15, 13 to be

$$
\rho(E)=1-\frac{\sin \left(\frac{\pi E}{\omega_{0}}\right)}{\left(\frac{\pi E}{\omega_{0}}\right)}
$$

This differs markedly from the clean result (2.6).

It is therefore challenging to find the crossover behavior between these two limits, and it might even be important for the transport problems associated with the excitations of quasiparticles inside the vortex core [14]. In order to achieve our goal, instead of treating the full Hamiltonian as random, we consider here only the scattering by impurities as a random matrix but not the full Ha miltonian.

In order to motivate the use of the symplectic structure in this problem it is instructive to examine the form of the matrix $A_{n m}^{i}$. For a given impurity $i$, let $A$ be the matrix whose elements are $\langle n|A| m\rangle=A_{n m}^{i}$. The state index $|m\rangle$ varies over $2 N$ levels. When $N=2$, for example, the states $\mid m>$ are $|-1>| 0>,,|1>| 2>$,. Since $E_{n}^{0}=-\omega_{0}\left(n-\frac{1}{2}\right)$, we have $E_{0}=-E_{1}=\frac{1}{2} \omega_{0}$ and $E_{2}=-E_{-1}=-\frac{3}{2} \omega_{0}$. Labelling the lines and rows of the matrix $A$ in the order $|0>| 2>,,|1>|-1>$,, in order to split it into even-even, even-odd, odd-odd $2 \times 2$ blocks, we write

$$
A=\left(\begin{array}{cccc}
<0|A| 0> & <0|A| 2> & <0|A| 1> & <0|A|-1> \\
<2|A| 0> & <2|A| 2> & <2|A| 1> & <2|A|-1> \\
<1|A| 0> & <1|A| 2> & <1|A| 1> & <1|A|-1> \\
<-1|A| 0> & <-1|A| 2> & <-1|A| 1> & <-1|A|-1>
\end{array}\right)
$$

Since $<0|A| 2>=A_{02}^{i}=A_{20}^{i}=<2|A| 0>$, and $<2|A| 1>=<0|A|-1>$, etc. from (2.4) (we have used the $\left.J_{-n}(x)=(-1)^{n} J_{n}(x)\right)$, it is easy to see that the matrix $A$ has, for arbitrary $N$, the structure

$$
A=\left(\begin{array}{cc}
a & b \\
b^{*} & -a^{T}
\end{array}\right)
$$

where $a$ is an $N \times N$ hermitian matrix $a^{\dagger}=a$, and $b$ is an $N \times N$ symmetric complex matrix $b^{T}=b\left(b^{T}\right.$ is the transpose of $\left.b\right)$. 
The number of degrees of freedom in the random matrix $A$ is thus $2 N^{2}+N,\left(N^{2}\right.$ for the hermitian matrix $a$ and $N^{2}+N$ for the complex symmetric matrix $b$ ). The number of generators of the symplectic $S p(N)$ group is indeed also $N(2 N+1)$.

The symplectic structure of $A$ is exhibited by the algebraic relation

$$
A^{T} J+J A=0
$$

where $J$ is

$$
J=\left(\begin{array}{cc}
0 & 1 \\
-1 & 0
\end{array}\right)
$$

This structure implies that the eigenvalues are real and pairwise opposite, giving a chiral structure to the eigenvalues. The Lie algebra $X$ is diagonalized by the symplectic group $G \in S p(N)$. For example, for $N=2$, we have

$$
X=G^{\dagger}\left(\begin{array}{cccc}
\lambda_{1} & & & \\
& \lambda_{2} & & \\
& & -\lambda_{1} & \\
& & & -\lambda_{2}
\end{array}\right) G
$$

If we mutiply the matrix $A$ by $i=\sqrt{-1}$ one recovers an element of the Lie algebra of the group $S p(N)$. If we consider now that the position $\left(\phi_{i}, r_{i}\right)$ of the impurity is random, it is natural to take $\langle n|A| m>$ in this $S p(N)$ invariant random ensemble.

For instance when there is only one impurity $(\mathrm{i}=1)$, the matrix $A$ has a periodic structure, with alternating signs for each 2 by 2 block. For example, in the case of $\mathrm{N}=4$, we have for the full Hamiltonian $H=E^{0}+A,\left(E^{0}\right.$ is a diagonal matrix $)$

$$
A=\left(\begin{array}{cccc}
s & -s & -c & c \\
-s & s & c & -c \\
-c & c & -s & s \\
c & -c & s & -s
\end{array}\right)
$$

where $s=\tilde{C} \sin \left(2 k_{F} a\right)$ and $c=\tilde{C} \cos \left(2 k_{F} a\right)$. ( In the single impurity case the phase $\phi$ can be set equal to zero by a choice of gauge condition ). This even-odd structure has been studied earlier in 12,11]. The periodic structure becomes clearer if we write the matrix $A=\left(A_{n m}\right)$, (2.4) for $\mathrm{N}=2$ with the approximation (2.5),

$$
A=\left(\begin{array}{cccc}
s & c & -s & -c \\
c & -s & -c & s \\
-s & -c & s & c \\
-c & s & c & -s
\end{array}\right)
$$

in which rows and lines are in the order $|2>| 1>,,|0>|-1>$,. The determinant of this matrix is a kind of Toeplitz determinant (constant along parallel to the anti-diagonal). We have omitted the diagonal part $E_{n}^{0}$. It is clear that the eigenvalues are periodic, depending on the odd-even parity of $n$. When we consider several impurities, a sum over impurities $\sum_{i} A_{n m}^{i}$ should be taken. In the clean limit, we replace the quantities $s$ and $c$ by $s=\sum_{i} \tilde{C} \sin \left(2 k_{F} r_{i}\right)$ and $c=\sum_{i} \tilde{C} \cos \left(2 k_{F} r_{i}\right)$. The random average over the positions of these impurities gives the density of state (2.7) [11]. 


\section{ITZYKSON-ZUBER INTEGRAL AND ITS EXTENSION}

In the previous section, we have discussed how we are led to study the crossover from the clean case to the dirty case. We now divide the matrix $\sum_{i=1}^{N_{i}} A_{n m}^{i}$ into two parts. One part, denoted by $H_{0}$, corresponds to the clean case, namely the matrix has a periodic structure as in (2.13), like a Toeplitz matrix, but with a sum over $i$. The other part is the remaining difference between the matrix $\sum_{i=1}^{N_{i}} A_{n m}^{i}$ and $H_{0}$, which is considered as a random matrix $V$. Thus we have

$$
H=H_{0}+V
$$

The matrices $H_{0}$ and $V$ are both symplectic, represented by the symplectic group Lie algebra $S p(N)$, which has the form (2.9).

In the analogous, but simpler, problem of a unitary invariant random perturbation, $\left(H_{0}\right.$ and $V$ were then both complex Hermitian matrices), a technique to study the crossover from the non-stochastic $H_{0}$ to a pure random matrix $V$ has been developped earlier [2 4 . Here we have to consider the symplectic case. As in the unitary case, we have to integrate over the Lie group which diagonalizes the matrix $H$. In the unitary case, the first step, the integration over the unitary group, was introduced in the study of two coupled random matrices by Itzykson and Zuber. [7]. We now summarize the formulae of integration over Lie Groups, which are useful for the subsequent discussion. We assume that the probability distribution of $V$ is a Gaussian, and that $H_{0}$ is a fixed non-random matrix. [It is possible to generalize it to some non-Gaussian distributions for $V$; for instance in the appendix B, we have considered the case of a general hypergeometric function whose argument is a matrix.]

The Itzykson-Zuber integral [7] is an integral over the unitary group $U(n)$,

$$
\int_{U(n)} e^{\operatorname{tr}\left(u a u^{\dagger} b\right)} d u=C_{N} \frac{\operatorname{det}\left(e^{a_{i} b_{j}}\right)}{\Delta(a) \Delta(b)}
$$

where $\Delta(a)=\prod_{i<j}\left(a_{i}-a_{j}\right)$, and similarly $\Delta(b)$, are the Vandermonde determinants of the eigenvalues of the Hermitian matrices $a$ and $b$. The constant is found to be $C_{N}=\prod_{j=1}^{N}(j-1)$ !. By expressing $\operatorname{det}\left(e^{a_{i} b_{j}}\right)$ as an alternating sum over the symmetric group $S_{n}$, one may write

$$
\int_{U(n)} e^{<\operatorname{Ad}(u) \cdot a \mid b>} d u=C_{N} \frac{\sum \epsilon(w) e^{<w \cdot a \mid b>}}{\Delta(a) \Delta(b)}
$$

where $\operatorname{Ad}(u) \cdot a=u a u^{-1}$ is the adjoint action of $u \in U(n)$ on the matrix $a$. In this formula the matrix $a$ is diagonal, $a=\operatorname{diag}\left(a_{1}, \ldots, a_{n}\right), w$ is a permutation and $\epsilon(w)$ is its signature. We have used the notation $\langle a \mid b\rangle=\operatorname{tr}(a b)$.

This formula is a special case of a more general formula due to Harish-Chandra [6]. Let $G$ be a compact connected Lie group. Then Harish-Chandra's result reads [6,17, 18,

$$
\int_{G} e^{<\operatorname{Ad}(g) \cdot a \mid b>} d g=\frac{\sum_{w \in W}(\operatorname{det} w) e^{<w \cdot a \mid b>}}{\Delta(a) \Delta(b)}
$$

where $a, b, h$ belong to a Lie algebra $h$, and for any $H \in h$, 


$$
\Delta(H)=\prod_{\alpha \in \Delta_{+}} \alpha(H)
$$

$\Delta_{+}$is the collection of the positive roots, $w$ is the finite reflection group, called the Weyl (or Coxeter) group and $h$ is the Cartan subalgebra.

The Harish-Chandra formula has been interpreted more recently as an integration over an orbit, with a symplectic structure. This structure implies that the saddle point method is exact, provided one sums over all the critical points [8, 19]. This is also related to the localization theorems 20].

As an illustration of the semi-classical nature of this formula, we consider again the integral over the unitary goup $U(N)$,

$$
I=\int \mathrm{d} g e^{\operatorname{tr}\left(a g b g^{\dagger}\right)}
$$

Representing an element $g$ of $U(N)$ as

$$
g=g_{0} e^{i X}
$$

where $X$ is an element of the Lie algebra, we expand the argument of the exponential. up to second order in $X$, and impose the stationarity condition,

$$
\operatorname{tr}\left[a g_{0} i X b g_{0}^{\dagger}\right]+\operatorname{tr}\left[a g_{0} b(-i X) g_{0}^{\dagger}\right]=0
$$

for any $X$, i.e.

$$
\left[b, g_{0}^{\dagger} a g_{0}\right]=0
$$

If $a$ and $b$ are diagonal, it implies that $g_{0}$ should be a unitary permutation matrix, $g_{0}=p$. Then, the integral $I$ becomes

$$
I=\sum_{p} e^{\operatorname{tr}\left(a p b p^{-1}\right)} \int d X \exp \left[\operatorname{tr}\left(-\frac{1}{2} a p X^{2} b p^{-1}-\frac{1}{2} a p b X^{2} p^{-1}+a p X b X p\right)\right]
$$

Performing the Gaussian integral over $X$ one recovers the Itzykson-Zuber formula. (This is not a derivation of course, but a way of verifying that for this integral the one-loop approximation is exact provided one sums over all the saddle-points).

A similar saddle point technique may be applied to the symplectic case. We take $g \in S p(N)$ in (3.6) and for $a$ and $b$ diagonal matrices with chiral eigenvalues, $a=$ $\operatorname{diag}\left(a_{1}, \ldots, a_{N},-a_{1}, \ldots,-a_{N}\right)$ and similarly for $b$. Then the same calculation yields

$$
I=C \frac{\operatorname{det}\left[2 \sinh \left(2 a_{i} b_{j}\right)\right]}{\prod_{1 \leq i<j \leq N}\left(a_{i}^{2}-a_{j}^{2}\right)\left(b_{i}^{2}-b_{j}^{2}\right) \prod_{1 \leq k \leq N}\left(a_{k} b_{k}\right)}
$$

and this result will be repeatedly used below. 


\section{DENSITY OF STATE}

Using the method developped for the unitary case [2 [5,9], we consider now the density of states with an external source matrix. Indeed for a random Gaussian $V$, the resulting probability for $H$ is a Gaussian with an external matrix source $A$ linearly coupled to $V$. We assume that the matrix $A$ belongs to the Lie algebra of the symplectic group and, without a loss of generality, we may take it as a diagonal matrix, $A=\operatorname{diag}\left(a_{1}, \ldots, a_{N},-a_{1}, \ldots,-a_{N}\right)$. We take this $A$ as the unperturbed $H_{0}$. The probability distribution of the random matrix $V$ is assumed to be Gaussian.

The density of states $\rho(\lambda)$ is given by

$$
\rho(\lambda)=\frac{1}{N}<\sum_{\alpha=1}^{N} \delta\left(\lambda-\lambda_{\alpha}\right)>
$$

The probability distribution $P(M)$ of the random matrix $M$ is

$$
\begin{aligned}
P(M) & =\exp \left[-\operatorname{tr} M^{2}-\operatorname{tr} M A\right] \\
& =\exp \left[-\sum \lambda_{i}^{2}-\operatorname{tr}\left(g \Lambda g^{\dagger} A\right)\right]
\end{aligned}
$$

where $\Lambda=\operatorname{diag}\left(\lambda_{1}, \ldots, \lambda_{N},-\lambda_{1}, \ldots,-\lambda_{N}\right)$ and $g$ is an element of the symplectic group. (Note that we use here a normalization which differs from our previous treatment [2, 1,5] of the unitary case). With the present normalization the edge of the density of states becomes of order $\sqrt{N}$; in the large $\mathrm{N}$ limit, the support of the density of states lies in an interval of order $[-\sqrt{2 N}, \sqrt{2 N}]$.

The density of states is obtained as the Fourier transform of the evolution operator

$$
\rho(\lambda)=\int_{-\infty}^{\infty} \frac{d t}{2 \pi} e^{-i t \lambda} U_{A}(t)
$$

and using the symplectic measure $\Delta^{2}(\lambda)$ with $\Delta(\lambda)=\prod\left(\lambda_{i}^{2}-\lambda_{j}^{2}\right) \prod \lambda_{k}$ [21], and the previous Harish-Chandra integral formula (3.11), we obtain

$$
\begin{aligned}
U_{A}(t) & =\frac{1}{N} \sum_{\alpha=1}^{N} \int_{0}^{\infty} \prod_{i=1}^{N} \mathrm{~d} \lambda_{\mathrm{i}} \frac{\prod_{1 \leq i<j \leq N}\left(\lambda_{i}^{2}-\lambda_{j}^{2}\right) \prod_{1 \leq k \leq N} \lambda_{k}}{\prod_{1 \leq i<j \leq N}\left(a_{i}^{2}-a_{j}^{2}\right) \prod_{1 \leq k \leq N} a_{k}} e^{-\sum \lambda_{i}^{2}+i t \lambda_{\alpha}} \\
& \times \operatorname{det}\left[\operatorname{sh}\left(2 \lambda_{i} a_{j}\right)\right]
\end{aligned}
$$

(up to a factor 2 in (3.11) absorbed in the coefficient $C$ ).

By the reflexion symmetry $\lambda_{i} \rightarrow-\lambda_{i}$, and by the exchange symmetry between $\lambda_{i}$ and $\lambda_{j}$, we can extend the integrations over the $\lambda_{i}$ 's from $-\infty$ to $\infty$. The change of sign for $i t \lambda_{i}$ can be absorbed since we can change $t \rightarrow-t$ by parity. Then the expression for $U_{A}(t)$ simplifies to

$$
U_{A}(t)=\frac{1}{N} \sum_{\alpha=1}^{N} \int_{-\infty}^{\infty} \prod_{i=1}^{N} \mathrm{~d} \lambda_{i} \frac{\prod_{1 \leq i<j \leq N}\left(\lambda_{i}^{2}-\lambda_{j}^{2}\right) \prod_{1 \leq k \leq N} \lambda_{k}}{\prod_{1 \leq i<j \leq N}\left(a_{i}^{2}-a_{j}^{2}\right) \prod_{1 \leq k \leq N} a_{k}} e^{-\sum \lambda_{i}^{2}+i t \lambda_{\alpha}+2 \sum_{i}^{N} a_{i} \lambda_{i}}
$$

Before proceeding to the explicit integrations over the $\lambda_{i}$ 's in $U_{A}(t)$, let us investigate the expression for the density of states $\rho(\lambda)$, in the absence of any external source, which 
one may then calculate by the usual method with orthogonal polynomials . The N-point level distribution is given

$$
\rho_{N}\left(\lambda_{1}, \ldots, \lambda_{N}\right)=C \prod_{1 \leq i<j \leq N}\left(\lambda_{i}^{2}-\lambda_{j}^{2}\right)^{2} \prod_{1 \leq k \leq N} \lambda_{k}^{2} \exp \left[-\sum \lambda_{i}^{2}\right]
$$

Using orthogonal polynomials method [10], this distribution may be written as

$$
\rho_{N}\left(\lambda_{1}, \ldots, \lambda_{N}\right)=\operatorname{det}\left[K_{N}\left(\lambda_{i}, \lambda_{j}\right)\right]
$$

with the kernel $K_{N}\left(\lambda_{i}, \lambda_{j}\right)$ given by a sum of Laguerre polynomials, and in particular the density of states $\rho(\lambda)=K_{N}(\lambda, \lambda)$ is equal to

$$
\rho(\lambda)=\frac{1}{N} \sum_{n=0}^{N-1} \frac{n !}{\Gamma\left(\frac{3}{2}+n\right)} L_{n}^{\left(\frac{1}{2}\right)}\left(\lambda^{2}\right) L_{n}^{\left(\frac{1}{2}\right)}\left(\lambda^{2}\right) \lambda^{2} e^{-\lambda^{2}} .
$$

$L_{n}^{(1 / 2)}(x)$ are associated (generalized) Laguerre polynomials, orthogonal with the normalization

$$
\int_{0}^{\infty} e^{-x} x^{\frac{1}{2}} L_{n}^{\left(\frac{1}{2}\right)}(x) L_{m}^{\left(\frac{1}{2}\right)}(x) d x=\frac{\Gamma\left(\frac{3}{2}+n\right)}{n !} \delta_{n, m}
$$

leading to $L_{0}^{(1 / 2)}(x)=1, L_{1}^{(1 / 2)}(x)=3 / 2-x, L_{n}^{(1 / 2)}(x)=\sum_{r=0}^{n}(-1)^{r}[\Gamma(n+3 / 2) / \Gamma(n-r+$ 1) $\Gamma(r+3 / 2)] x^{r} / r !$.

The asmptotic behavior of these Laguerre polynomials in the limit $N \rightarrow \infty$ is [23]

$$
L_{N}^{\left(\frac{1}{2}\right)}(x)=\frac{1}{\sqrt{\pi x}} e^{\frac{1}{2} x} \sin (2 \sqrt{N x})+O\left(\frac{1}{\sqrt{N}}\right) .
$$

With the Christoffel-Darboux identity, we have

$$
\begin{aligned}
& \sum_{n=0}^{N-1} \frac{n !}{\Gamma\left(\frac{3}{2}+n\right)} L_{n}^{\left(\frac{1}{2}\right)}(x) L_{n}^{\left(\frac{1}{2}\right)}(y) \\
= & -\frac{N !}{\Gamma\left(\frac{1}{2}+N\right)} \frac{L_{N}^{\left(\frac{1}{2}\right)}(x) L_{N-1}^{\left(\frac{1}{2}\right)}(y)-L_{N-1}^{\left(\frac{1}{2}\right)}(x) L_{N}^{\left(\frac{1}{2}\right)}(y)}{x-y}
\end{aligned}
$$

and using the large $N$ aymptotic behavior (4.10), we obtain

$$
\begin{aligned}
& x e^{-x}\left[\sum_{n=0}^{N-1} \frac{n !}{\Gamma\left(\frac{3}{2}+n\right)} L_{n}^{\left(\frac{1}{2}\right)}(x) L_{n}^{\left(\frac{1}{2}\right)}(x)\right] \\
= & x e^{-x}\left[L_{N}^{\left(\frac{1}{2}\right)}(x) \frac{d}{d x} L_{N-1}^{\left(\frac{1}{2}\right)}(x)-L_{N-1}^{\left(\frac{1}{2}\right)}(x) \frac{d}{d x} L_{N}^{\left(\frac{1}{2}\right)}(x)\right] \\
\simeq & \frac{1}{\pi}\left[\frac{\sqrt{N}}{\sqrt{x}} \sin \left(\sqrt{\frac{x}{N}}\right)-\frac{1}{4 \sqrt{N x}} \sin (4 \sqrt{N x})\right] \\
\simeq & \frac{1}{\pi}\left[1-\frac{1}{4 \sqrt{N x}} \sin (4 \sqrt{N x})\right]
\end{aligned}
$$


Thus, putting $x=\lambda^{2}$, we get

$$
\rho(\lambda)=\frac{1}{\pi}\left[1-\frac{\sin (4 \sqrt{N} \lambda)}{4 \sqrt{N} \lambda}\right] .
$$

This result is identical to (2.7), when we substitute $\omega_{0}=\frac{\pi}{4 N}$ and $E=\lambda / \sqrt{N}$.

We now return to the integral (4.5) over the $\lambda_{i}$ 's in the presence of the external source. We first replace $t \rightarrow 2 t$ and following [2, 3], for fixed $\alpha$, we denote by $b_{i}$ the sum $b_{i}=a_{i}+i t \delta_{i, \alpha}$ . One then uses the integral

$$
\int_{-\infty}^{\infty} \mathrm{d} \lambda_{i} \prod_{i<j}\left(\lambda_{i}^{2}-\lambda_{j}^{2}\right) \prod_{i=1}^{N} \lambda_{i} e^{-\sum\left(\lambda_{i}-b_{i}\right)^{2}}=\prod_{i<j}\left(b_{i}^{2}-b_{j}^{2}\right) \prod_{i=1}^{N} b_{i}
$$

(after the translation $\lambda_{i} \rightarrow \lambda_{i}+b_{i}$ the result follows easily from antisymmetry under permutation of the $b_{i}$ 's, parity in $b_{i}$, and counting the degree of the resulting polynomial in those b's).

Using the normalization $U_{A}(0)=1$, and writing the sum over $\alpha$ as a contour integral around the $a_{j}^{2}$ 's in the complex $u$-plane, we obtain

$$
U_{A}(t)=\frac{1}{N} \oint \frac{d u}{2 \pi i} \prod_{j=1}^{N} \frac{\left((\sqrt{u}+i t)^{2}-a_{j}^{2}\right)}{\left(u-a_{j}^{2}\right)} \frac{1}{(\sqrt{u}+i t)^{2}-u}\left(1+\frac{i t}{\sqrt{u}}\right) e^{-t^{2}+2 i t \sqrt{u}}
$$

Fourier transforming $U_{A}(t)$ we obtain the density of states $\rho(\lambda)$

$$
\rho(\lambda)=\int_{-\infty}^{\infty} U_{A}(t) e^{-2 i t \lambda} \frac{d t}{2 \pi}
$$

Let us verify the consistency with the orthogonal polynomial result (4.8) when the external source vanishes. Then

$$
U_{0}(t)=\frac{1}{N} \oint \frac{d u}{2 \pi i} \frac{(\sqrt{u}+i t)^{2 N}}{u^{N}} \frac{1}{(\sqrt{u}+i t)^{2}-u}\left(1+\frac{i t}{\sqrt{u}}\right) e^{-t^{2}+2 i t \sqrt{u}}
$$

and

$$
\begin{aligned}
\rho(\lambda) & =\int_{-\infty}^{\infty} \frac{d t}{2 \pi} U_{0}(t) e^{-2 i t \lambda} \\
& =\frac{i(-1)^{N}}{N} \int_{-\infty}^{\infty} \frac{d x}{2 \pi} \oint \frac{d u}{2 \pi i}\left(\frac{x}{\sqrt{u}}\right)^{2 N+1} \frac{1}{u+x^{2}} e^{-x^{2}-2 i x \lambda-u+2 \lambda \sqrt{u}}
\end{aligned}
$$

(we have shifted $t$ to $t=x+i \sqrt{u}$ ). The integration contour is a small loop around the origin $u=0$. Expanding $1 /\left(u+x^{2}\right)=x^{-2}\left(1-\frac{u}{x^{2}}+\cdots\right)$, and noting that

$$
\oint \frac{d u}{2 \pi i} \frac{1}{u^{n+1} \sqrt{u}} e^{-u+2 \lambda \sqrt{u}}=\frac{2 \Gamma\left(\frac{3}{2}\right)}{\Gamma\left(\frac{3}{2}+n\right)}(-1)^{n} \lambda L_{n}^{\left(\frac{1}{2}\right)}\left(\lambda^{2}\right)
$$

and 


$$
\int_{-\infty}^{\infty} \frac{d x}{2 \pi} x^{2 n+1} e^{-x^{2}-2 i x \lambda}=-\frac{i n ! \lambda}{2 \sqrt{\pi}} L_{n}^{\left(\frac{1}{2}\right)}\left(\lambda^{2}\right) e^{-\lambda^{2}}
$$

we $\mathrm{v}$ erify the consistency of the integral representation (4.18) with the orthogonal polynomial result (4.8). The integral representation (4.20), gives an easy way to recover the large $n$ asymptotic behavior (4.11) of the Laguerre polynomials through the steepest descent method. Note that the chiral invariance leads as expected to an even density of states.

In the presence of the external source, the density of states becomes

$$
\rho(\lambda)=\frac{i(-1)^{N-1}}{N} \int_{-\infty}^{\infty} \frac{d x}{2 \pi} \oint \frac{d u}{2 \pi i}\left(\frac{x}{\sqrt{u}}\right) \prod_{\gamma=1}^{N}\left(\frac{x^{2}+a_{\gamma}^{2}}{u-a_{\gamma}^{2}}\right) \frac{1}{u+x^{2}} e^{-x^{2}-2 i x \lambda-u+2 \lambda \sqrt{u}}
$$

(We shall analyze this density of states as the limit of $\lambda \rightarrow \mu$ of the kernel $K_{N}(\lambda, \mu)$ in the next section). We note here that if we make the change of variable, $u=v^{2}$, we have

$$
\rho(\lambda)=\frac{i(-1)^{N-1}}{N} \int_{-\infty}^{\infty} \frac{d x}{2 \pi} \oint \frac{d v}{2 \pi i} \prod_{\gamma=1}^{N}\left(\frac{x^{2}+a_{\gamma}^{2}}{v^{2}-a_{\gamma}^{2}}\right) \frac{x}{v^{2}+x^{2}} e^{-x^{2}-2 i x \lambda-v^{2}+2 \lambda v}
$$

where the contour encloses all the $\pm a_{\gamma}$. For example when $N=1$, we obtain

$$
\begin{aligned}
\rho(\lambda) & =\frac{\lambda}{2 \sqrt{\pi}} \frac{\sinh \left(2 \lambda a_{1}\right)}{a_{1}} e^{-a_{1}^{2}-\lambda^{2}} \\
& =\frac{\lambda}{4 \sqrt{\pi} a_{1}}\left[e^{-\left(\lambda-a_{1}\right)^{2}}-e^{-\left(\lambda+a_{1}\right)^{2}}\right]
\end{aligned}
$$

Near the origin $\lambda=0$, the density of states $\rho(\lambda)$ becomes $\rho(\lambda) \sim \frac{\lambda^{2}}{\pi} \exp \left(-a_{1}^{2}-\lambda^{2}\right)$. Thus the density of states is an even function and it vanishes near the origin as $\lambda^{2}$.

\section{CORRELATON FUNCTION}

The $n$-level correlation functions are expressed as the determinant of the kernel $K_{N}(\lambda, \mu)$, exactly as for Hermitian random matrices in a source. Indeed the formula of (4.14) is quite similar to (3.8) of [5]. Therefore, all the derivations of the Hermitian matrix model in [2: [-.5], may be repeated almost identically for the $S p(N)$ case.

For instance, the two-level correlation function $\rho^{(2)}(\lambda, \mu)$ is obtained as the double Fourier transform of $U_{A}\left(t_{1}, t_{2}\right)$,

$$
\rho^{(2)}(\lambda, \mu)=\iint \frac{d t_{1} d t_{2}}{(2 \pi)^{2}} e^{-i t_{1} \lambda-i t_{2} \mu} U_{A}\left(t_{1}, t_{2}\right)
$$

in which $U_{A}\left(t_{1}, t_{2}\right)$ is defined as

$$
U_{A}\left(t_{1}, t_{2}\right)=<\frac{1}{N} \sum_{\alpha=1}^{N} e^{i t_{1} \lambda_{\alpha}} \frac{1}{N} \sum_{\beta=1} e^{i t_{2} \lambda_{\beta}}>
$$


Using the integral formulae (3.10) and (4.14), we find

$$
\begin{aligned}
U_{A}\left(t_{1}, t_{2}\right) & =\frac{1}{N^{2}} \sum_{\alpha \neq \beta} \frac{\left(a_{\alpha}+i t_{1}\right)^{2}-\left(a_{\beta}+i t_{2}\right)^{2}}{\left(a_{\alpha}^{2}-a_{\beta}^{2}\right)} \prod_{\gamma \neq(\alpha, \beta)} \frac{\left(a_{\alpha}+i t_{1}\right)^{2}-a_{\gamma}^{2}}{a_{\alpha}^{2}-a_{\gamma}^{2}} \\
& \times \prod_{\gamma \neq(\alpha, \beta)} \frac{\left(a_{\beta}+i t_{1}\right)^{2}-a_{\gamma}^{2}}{a_{\beta}^{2}-a_{\gamma}^{2}}\left(1+\frac{i t}{a_{\alpha}}\right)\left(1+\frac{i t_{2}}{a_{\beta}}\right) e^{-t_{1}^{2}-t_{2}^{2}+2 i \sqrt{u} t_{1}+2 i \sqrt{v} t_{2}}
\end{aligned}
$$

(wa have subtracted the term $\alpha=\beta$ in $U_{A}\left(t_{1}, t_{2}\right)$ ). The summation over $\alpha$ and $\beta$ is expressed by contour integration over two complex variables $u$ and $v$,

$$
\begin{aligned}
U_{A}\left(t_{1}, t_{2}\right) & =\frac{1}{N^{2}} \oint \frac{d u d v}{(2 \pi i)^{2}} \prod_{\gamma=1}^{N} \frac{\left(\sqrt{u}+i t_{1}\right)^{2}-a_{\gamma}^{2}}{\left(u-a_{\gamma}^{2}\right)} \prod_{\gamma=1}^{N} \frac{\left(\sqrt{v}+i t_{2}\right)^{2}-a_{\gamma}^{2}}{v-a_{\gamma}^{2}} \\
& \times \frac{1}{\left(\sqrt{u}+i t_{1}\right)^{2}-v} \frac{1}{\left(\sqrt{v}+i t_{2}\right)^{2}-u}\left(1+\frac{i t_{1}}{\sqrt{u}}\right)\left(1+\frac{i t_{2}}{\sqrt{v}}\right) \\
& \times \frac{(u-v)\left[\left(\sqrt{u}+i t_{1}\right)^{2}-\left(\sqrt{v}+i t_{2}\right)^{2}\right]}{\left[\left(\sqrt{u}+i t_{1}\right)^{2}-u\right]\left[\left(\sqrt{v}+i t_{2}\right)^{2}-v\right]} e^{-t_{1}^{2}-t_{2}^{2}+2 i \sqrt{u} t_{1}+2 i \sqrt{v} t_{2}}
\end{aligned}
$$

We now shift $t_{1} \rightarrow t_{1}+i \sqrt{u}$ and $t_{2} \rightarrow t_{2}+i \sqrt{v}$, note that

$$
\frac{(u-v)\left[\left(i t_{1}\right)^{2}-\left(i t_{2}\right)^{2}\right]}{\left[\left(i t_{1}\right)^{2}-v\right]\left[\left(i t_{2}\right)^{2}-u\right]}=\frac{\left[\left(i t_{1}\right)^{2}-u\right]\left[\left(i t_{2}\right)^{2}-v\right]}{\left[\left(i t_{1}\right)^{2}-v\right]\left[\left(i t_{2}\right)^{2}-u\right]}-1
$$

and divide $U_{A}\left(t_{1}, t_{2}\right)$ into two terms. The first one, which comes from $(-1)$ gives the product of the two density of states $\rho(\lambda) \rho(\mu)$, and the second one is a product of two integrals over $t_{1}, u$ and $t_{2}, v$ respectively. Therefore, we end up with

$$
\rho^{(2)}(\lambda, \mu)=K_{N}(\lambda, \lambda) K_{N}(\mu, \mu)-K_{N}(\lambda, \mu) K_{N}(\mu, \lambda)
$$

with

$$
\begin{aligned}
K_{N}(\lambda, \mu) & =\frac{1}{N} \oint \frac{d u}{2 \pi i} \int_{-\infty}^{\infty} \frac{d t}{2 \pi} e^{-t^{2}-2 i t \mu-u+2 \sqrt{u} \lambda} \prod_{\gamma=1}^{N} \frac{(i t)^{2}-a_{\gamma}^{2}}{u-a_{\gamma}^{2}} \frac{1}{(i t)^{2}-u} \\
& \times\left(\frac{i t}{\sqrt{u}}\right)
\end{aligned}
$$

(the contour in the $u$-plane encircles all the $a_{\gamma}^{2}$ ). From this expression, we recover the previous result (4.15,4.16) for the density of states as $\rho(\lambda)=K_{N}(\lambda, \lambda)$.

The n-point correlation can be analyzed as in [5] and leads here also to a determinant form,

$$
\rho\left(\lambda_{1}, \cdots, \lambda_{n}\right)=\operatorname{det}\left[K_{N}\left(\lambda_{i}, \lambda_{j}\right)\right]
$$

for an arbitrary external source. 
We now discuss Dyson's short-distance universality, within our model, i.e. when we vary the external source $A$, through the explicit expression of the kernel $K_{N}(\lambda, \mu)$.

We return first to the sourceless case, $a_{\gamma}=0$, and consider the large $\mathrm{N}$ limit of $K_{N}(\lambda, \mu)$ for $\lambda, \mu$ are order of one. We scale $t \rightarrow \sqrt{N} t$, and $u \rightarrow N u$. The kernel is then

$$
K_{N}(\lambda, \mu)=-\frac{1}{\sqrt{N}} \oint \frac{d u}{2 \pi i} \int \frac{d t}{2 \pi} e^{-N(f(t)+g(u))}\left(\frac{i t}{\sqrt{u}}\right) \frac{1}{t^{2}+u}
$$

where $f(t)=t^{2}+2 i \mu / \sqrt{N}-2 \ln t$ and $g(u)=u-2 \lambda \sqrt{u} / \sqrt{N}+\ln u$. In the large N-limit we have the saddle points, $t_{c}^{(1)}=1-i \mu / 2 \sqrt{N}, t_{c}^{(2)}=-1-i \mu / 2 \sqrt{N}, u_{c}^{(1)}=-1+i \lambda / 2 \sqrt{N}$ and $u_{c}^{(2)}=-1-i \mu / 2 \sqrt{N}$. Adding these saddle-points contributions, we obtain

$$
K_{N}(\lambda, \mu)=\frac{1}{\pi}\left[\frac{\sin (2 \sqrt{N}(\lambda-\mu))}{2 \sqrt{N}(\lambda-\mu)}-\frac{\sin (2 \sqrt{N}(\lambda+\mu))}{2 \sqrt{N}(\lambda+\mu)}\right]
$$

When $\lambda \rightarrow \mu$, we recover the expression (4.13) of the density of states.

In the presence of the external source, we find a similar expression to Eq.(5.9), with

$$
\begin{aligned}
& f(t)=t^{2}+\frac{2 i t \mu}{\sqrt{N}}-\frac{1}{N} \sum \ln \left(t^{2}+\frac{a_{\gamma}^{2}}{N}\right) \\
& g(u)=u-\frac{2 \lambda \sqrt{u}}{\sqrt{N}}+\frac{1}{N} \sum \ln \left(u-\frac{a_{\gamma}^{2}}{N}\right)
\end{aligned}
$$

Using the definition $, f^{\prime}\left(t_{c}\right)=0, g^{\prime}\left(u_{c}\right)=0$, of the saddle-points we find

$$
K_{N}(\lambda, \mu)=\frac{1}{\pi}\left[\frac{\sin \left(2 t_{c 0} \sqrt{N}(\lambda-\mu)\right)}{2 \sqrt{N}(\lambda-\mu)}-\frac{\sin \left(2 t_{c 0} \sqrt{N}(\lambda+\mu)\right)}{2 \sqrt{N}(\lambda+\mu)}\right]
$$

where $t_{c 0}$ is a solution of

$$
\frac{1}{N} \sum_{\gamma=1}^{N} \frac{1}{t_{c 0}^{2}+\frac{a_{\gamma}^{2}}{N}}=1
$$

Here we have assumed that the order of $a_{\gamma}$ is $a_{\gamma}^{2} \leq O(N)$. The derivation of (5.13) is the same as (5.10). The difference is just a normalization, due to the change of the saddle-point $t_{c 0}$. Note that the support of the density of states is inside the interval of $-\sqrt{N}$ and $\sqrt{N}$. Therefore, universality holds provided that the eigenvalues of the external source matrix are located in an interval of the same order of magnitude ; (it has to be of same order as the support of the density of states for the zero external source case). In this universal regime for the correlation function, the energies $\lambda$ and $\mu$ are assumed of order one, namely $\lambda / \sqrt{N} \rightarrow 0$ in the large $N$ limit. Since $\lambda$ can be order of $\sqrt{N}$, the universal behavior of (5.13) appears only near the origin, as exepcted, and is differnt from the non-universal bulk behaviour. The universality found here, is similar to that found in other chiral random matrix models studied in [3]; there it was the density of states which took a universal form near the origin, 
and became independent of the external source or of the non-Gaussian distributions (as seen by a rescaling of the energy).

When the $a_{\gamma}^{2}$ spread over an interval larger than $N$, the universal form of (5.13) does not hold any more, since the saddle-point method of (5.14) breaks down. In our formulation, the amplitude of the random matrix is fixed. We now change the strength of the external source. For this purpose, we introduce a parameter $C$, which determines the strength of the external source $H_{0}$, respective to the random potential $V$. Let us consider the example of the external source,

$$
a_{\gamma}=C \tan \left[\frac{(2 \gamma-1) \pi}{2(2 N+1)}\right]
$$

where $\gamma=1,2, \ldots, N$, and $C$ is a parameter. When $\gamma /(2 N+1)<<1$, we have

$$
a_{\gamma}=\frac{C \pi}{(2 N+1)}\left(\gamma-\frac{1}{2}\right)
$$

and the eigenvalues of the external source are equally distributed, and thus reproduce the excitation spectrum inside the clean superconductor vortex, if we identify $\hbar \omega_{0}=C \pi /(2 N+1)$ in (2.3). Since we are interested in the behavior near the origin for the energies $\lambda$ and $\mu$ in $K_{N}(\lambda, \mu)$, this linear approximation of the $\tan (x) \simeq x$ is valid.

More generally, using the formula,

$$
\prod_{m=1}^{N}\left(x^{2}+\tan ^{2}\left[\frac{(2 m-1) \pi}{2(2 N+1)}\right]\right)=\frac{1}{2(2 N+1)}\left[(1+x)^{2 N+1}+(1-x)^{2 N+1}\right]
$$

we may replace the product involving the $a_{\gamma}$ in (5.7), and obtain

$$
\begin{aligned}
K_{N}(\lambda, \mu) & =-\frac{1}{N} \oint \frac{d u}{2 \pi i} \int \frac{d t}{2 \pi}\left[\frac{\left(1+\frac{t}{C}\right)^{2 N+1}+\left(1-\frac{t}{C}\right)^{2 N+1}}{\left(1+\frac{i \sqrt{u}}{C}\right)^{2 N+1}+\left(1-\frac{i \sqrt{u}}{C}\right)^{2 N+1}}\right] \\
& \times \frac{1}{t^{2}+u}\left(\frac{i t}{\sqrt{u}}\right) e^{-t^{2}-2 i t \mu-u+2 \sqrt{u} \lambda}
\end{aligned}
$$

This expression is exact for finite $N$ when the $a_{\gamma}$ are given by (5.15). It is easily seen that when $C \rightarrow 0$, we recover the previous external source free result (5.9).

For non-zero $C$ we have two different cases according to the size of $C$,

i) if $C$ is of order $\sqrt{N}$, we recover the universal behavior of (5.13). (We simply scale $t \rightarrow \sqrt{N} t$, and $u \rightarrow N u$, and use the saddle point method). We recover then the result (5.13), with

$$
t_{c 0}=\frac{1}{2}\left(\sqrt{\frac{C^{2}}{N}+4}-\frac{C}{\sqrt{N}}\right)
$$

ii) in the second case $C \sim O(N)$, we change $u=v^{2}$ in (5.18) :

$$
\begin{aligned}
K_{N}(\lambda, \mu) & =-\frac{1}{N} \oint \frac{d v}{2 \pi i} \int \frac{d t}{2 \pi}\left[\frac{\left(1+\frac{t}{C}\right)^{2 N+1}+\left(1-\frac{t}{C}\right)^{2 N+1}}{\left(1+\frac{i v}{C}\right)^{2 N+1}+\left(1-\frac{i v}{C}\right)^{2 N+1}}\right] \\
& \times \frac{i t}{t^{2}+v} e^{-t^{2}-2 i t \mu-v^{2}+2 v \lambda}
\end{aligned}
$$


In the large N-limit (5.20) reduces to

$$
\begin{aligned}
K_{N}(\lambda, \mu) & =-\frac{1}{N} \oint \frac{d v}{2 \pi i} \int \frac{d t}{2 \pi} \frac{\cosh \left(\frac{2 N t}{C}\right)}{\cos \left(\frac{2 N v}{C}\right)} \frac{i t}{t^{2}+v^{2}} \\
& \times e^{-t^{2}-2 i t \mu-v^{2}+2 v \lambda}
\end{aligned}
$$

If we rescale $t \rightarrow \sqrt{N} t$ and $v \rightarrow \sqrt{N} v$ poles appears in the $v$-plane at $v=C\left(m-\frac{1}{2}\right) \pi / 2 N^{3 / 2}$ for which $\cos \left(2 N^{3 / 2} v / C\right)=0 ; m$ is an integer.

Within the residues of these poles, we have the Gaussian factors

$$
e^{-N\left(v-\frac{\lambda}{\sqrt{N}}\right)^{2}}=e^{-\left(\frac{C\left(m-\frac{1}{2}\right) \pi}{2 N}-\lambda\right)^{2}}
$$

There are thus two different regions for $\lambda$. We first consider $\lambda \sim O(\sqrt{N})$, i.e. the bulk case. Then $\lambda / \sqrt{N}>>\sqrt{N} / C$, and the saddle point of $t$ is $t_{0}=-i \lambda / \sqrt{N}$. We set $\lambda=\mu$, and for this this saddle point, we have the density of states $\rho(\lambda)=K_{N}(\lambda, \lambda)$

$$
\begin{aligned}
\rho(\lambda) & =-\frac{1}{2 \sqrt{\pi N}} \sum_{m}(-1)^{m}\left[\frac{\lambda \cos \left(\frac{2 N \lambda}{C}\right)}{-\lambda^{2}+\left(\frac{C\left(m-\frac{1}{2}\right) \pi}{2 N}\right)^{2}}\right] \\
& \times e^{-\left(\frac{C\left(m-\frac{1}{2}\right) \pi}{2 N}-\lambda\right)^{2}}
\end{aligned}
$$

Note that the zeros of the denominator are cancelled by zeros of the numerator $\cos (2 N \lambda / C)$. When $C / N>>1$, the exponential factor of (5.23) damps the result and we may set $\lambda=C(m-1 / 2) \pi /(2 N)$, then

$$
\frac{\lambda \cos \left(\frac{2 N \lambda}{C}\right)}{-\lambda^{2}+\left(\frac{C\left(m-\frac{1}{2}\right) \pi}{2 N}\right)^{2}}=-(-1)^{m} \frac{N}{C}
$$

Thus we find

$$
\rho(\lambda)=\frac{\sqrt{N}}{2 C \sqrt{\pi}} \sum_{m} e^{-\left(\frac{C\left(m-\frac{1}{2}\right) \pi}{2 N}-\lambda\right)^{2}}
$$

which is a sum of Gaussians : the density of states is just sum of Gaussian peaks around the levels $\lambda=C(m-1 / 2) \pi / 2 N$. This result is valid provided $\lambda$ is not too close to the origin.

When $\lambda$ is near the origin, namely $\lambda \sim O(1)$, we have $\lambda / \sqrt{N} \sim \sqrt{N} / C$ since $C$ is order $N$. In this case, we have to take into account the term $\cosh (2 N t / C)$ in the saddle-point equation. The saddle-point becomes $t_{0}=-i \lambda / \sqrt{N} \pm \sqrt{N} / C$. Using this value in (5.21), we find

$$
\rho(\lambda)=\operatorname{Re} \sum(-1)^{n}\left[\frac{\lambda+i \frac{N}{C}}{-\left(\lambda+i \frac{N}{C}\right)^{2}+\left(\frac{C}{2 N}\left(n-\frac{1}{2}\right) \pi\right)^{2}}\right] e^{-\left[\frac{C}{2 N}\left(n-\frac{1}{2}\right) \pi-\lambda\right]^{2}}
$$

Near $\lambda=0$, the density of states behaves as $\rho(\lambda) \sim \lambda^{2}$. For example, when $C=N$, we have $\rho(\lambda) \sim \pi \lambda^{2} \exp \left(-\pi^{2} / 16\right) /\left(1+\pi^{2} / 16\right)$, and $\rho(\lambda)$ has peaks at $\lambda= \pm \pi / 4, \pm 3 \pi / 4, \ldots$ Thus, when $C \sim N$, which means that the random potential is weak compared to the external source, the density of states takes a form which is different from the universal form (5.13). 


\section{CROSSOVER FROM THE CLEAN LIMIT TO THE DIRTY LIMIT}

In the clean case, when $k_{F} r_{i} \sim k_{F} \xi>>1$, we approximate $A_{n m}^{i}$ by (2.12). Then, the periodic spectrum is obtained

$$
E_{n}=-\hbar \omega_{0}\left(n-\frac{1}{2}+\tilde{z}(-1)^{n}\right)
$$

When the number of impurities inside the vortex $N_{i} \simeq 1$, we are in the superclean case, and $\tilde{z}$ in (6.1) is a function of the position of this impurity. If $1<N_{i}<N_{i c}$, we are in a clean case, and we need to average over the different values of $\tilde{z}$.

In the previous section, we have discussed the case where the deterministic term $E_{n}=$ $-\hbar \omega\left(n-\frac{1}{2}\right)$ is coupled to the random matrix $A$. Here we consider for the external source the matrix whose eigenvalues are (6.1).

We may then use the formula

$$
\prod_{r=1}^{N}\left[x^{2}+\tan ^{2}\left(\frac{r \pi}{2(N+1)}\right)\right]=\frac{1}{4(N+1) x}\left[(1+x)^{2(N+1)}-(1-x)^{2(N+1)}\right],
$$

which is similar to (5.17). ( We assume that $N$ is odd here). We divide this product into two parts, r-odd and r-even . The r-even part is obtained immediately from (6.2) as

$$
\prod_{r=\text { even }}^{N-1}\left[x^{2}+\tan ^{2}\left(\frac{r \pi}{2(N+1)}\right)\right]=\frac{1}{2(N+1) x}\left[(1+x)^{N+1}-(1-x)^{N+1}\right]
$$

Dividing (6.2) by (6.3), we obtain the expression for the r-odd part,

$$
\prod_{r=o d d}^{N}\left[x^{2}+\tan ^{2}\left(\frac{r \pi}{2(N+1)}\right)\right]=\frac{1}{2}\left[(1+x)^{N+1}+(1-x)^{N+1}\right]
$$

We now consider the eigenvalues 6.1) as an external source,

$$
a_{\gamma}=[C \pi / 2(N+1)]\left(\gamma-\frac{1}{2}+\tilde{z}(-1)^{\gamma}\right)
$$

and introduce $z_{0}=C \pi / 4(N+1), z=-C \pi \tilde{z} / 2(N+1)$. Using the expressions $(6.3)$ and (6.4), we write

$$
\begin{aligned}
& \left(\frac{x-i z_{0}-i z}{C}\right) \prod_{r=\text { even }}^{N-1}\left[\left(\frac{x-i z_{0}-i z}{C}\right)^{2}+\tan ^{2}\left(\frac{r \pi}{2(N+1)}\right)\right] \\
\times & \prod_{r=\text { odd }}^{N}\left[\left(\frac{x-z_{0}+z}{C}\right)^{2}+\tan ^{2}\left(\frac{r \pi}{2(N+1)}\right)\right] \\
= & \frac{1}{4(N+1)}\left[\left(1+\frac{x-i z_{0}-i z}{C}\right)^{N+1}-\left(1-\frac{x-i z_{0}-i z}{C}\right)^{N+1}\right] \\
\times & {\left[\left(1+\frac{x-i z_{0}+i z}{C}\right)^{N+1}+\left(1+\frac{x-i z_{0}+i z}{C}\right)^{N+1}\right] }
\end{aligned}
$$


In the large $\mathrm{N}$ limit, when $r \pi /(2(N+1))<<1$, the left hand side of (6.5) vanishes at $x=-i\left( \pm r-1 / 2+z(-1)^{r}\right) C \pi /[2(N+1)]$. Noting that

$$
\begin{aligned}
\prod_{\gamma}\left(t^{2}+a_{\gamma}^{2}\right) & =\prod_{\gamma}\left[t-i\left(z_{0}+z\right)+i \frac{C \gamma \pi}{2(N+1)}\right]\left[t-i\left(z_{0}+z\right)-i \frac{C \gamma \pi}{2(N+1)}\right] \\
& =\prod_{\gamma}\left[\left(t-i\left(z_{0}+z\right)\right)^{2}+C^{2} \tan ^{2}\left(\frac{\gamma \pi}{2(N+1)}\right)\right]
\end{aligned}
$$

we have from (5.7) (and the change of variable $\sqrt{u}=v$ ),

$$
\begin{aligned}
K_{N}(\lambda, \mu) & =-\frac{1}{N} \oint \frac{d v}{2 \pi i} \int \frac{d t}{2 \pi} e^{-t^{2}-2 i t \mu-v^{2}+2 v \lambda} \frac{i t}{t^{2}+v^{2}} \\
& \times \frac{\left[\left(1+\frac{i}{C}\left(-i t-z_{0}-z\right)\right)^{N+1}-\left(1-\frac{i}{C}\left(-i t-z_{0}-z\right)\right)^{N+1}\right]}{\left[\left(1+\frac{i}{C}\left(v-z_{0}-z\right)\right)^{N+1}-\left(1-\frac{i}{C}\left(v-z_{0}-z\right)\right)^{N+1}\right]} \\
& \times \frac{\left[\left(1+\frac{i}{C}\left(-i t-z_{0}+z\right)\right)^{N+1}+\left(1-\frac{i}{C}\left(-i t-z_{0}+z\right)\right)^{N+1}\right]}{\left[\left(1+\frac{i}{C}\left(v-z_{0}+z\right)\right)^{N+1}+\left(1-\frac{i}{C}\left(v-z_{0}+z\right)\right)^{N+1}\right]}
\end{aligned}
$$

As in the previous section, we have to distinguish two different cases, i) $C \sim O(N)$ and ii) $C \sim O(\sqrt{N})$. There are also two regions $\lambda \sim O(\sqrt{N})$ and $\lambda \sim O(1)$.

When $C \sim O(N)$, we exponentiate the factor in the bracket of (6.7),

$$
\begin{aligned}
& \frac{\sin \left[\frac{N}{C}\left(-i t-z_{0}-z\right)\right] \cos \left[\frac{N}{C}\left(-i t-z_{0}+z\right)\right]}{\sin \left[\frac{N}{C}\left(v-z_{0}-z\right)\right] \cos \left[\frac{N}{C}\left(v-z_{0}+z\right)\right]} \\
= & \frac{\sin \left[\frac{2 N}{C}\left(-i t-z_{0}\right)\right]-\sin \left(\frac{2 N}{C} z\right)}{\sin \left[\frac{2 N}{C}\left(v-z_{0}\right)\right]-\sin \left(\frac{2 N}{C} z\right)}
\end{aligned}
$$

Since $z_{0}=C \pi /[4(N+1)]$, we have

$$
\begin{aligned}
K_{N}(\lambda, \mu) & =-\frac{1}{N} \oint \frac{d v}{2 \pi i} \int \frac{d t}{2 \pi} \frac{\cosh \left(\frac{2 N t}{C}\right)+\sin \left(\frac{2 N}{C} z\right)}{\cos \left(\frac{2 N v}{C}\right)+\sin \left(\frac{2 N}{C} z\right)} \frac{i t}{t^{2}+v^{2}} \\
& \times e^{-t^{2}-2 i t \mu-v^{2}+2 v \lambda}
\end{aligned}
$$

When $z=0$, we obtain the same expression as in the previous section (5.21).

Poles in the integral over $v$ are present at $v=z_{0}+z+C n \pi / N$ and $v=z_{0}-z+(2 n+$ 1) $(C \pi / 2 N)$ from (6.8). The residues $R$ for the first poles are

$$
R=(-1)^{n} i t \frac{\sin \left[\frac{N}{C}\left(-i t-z_{0}-z\right)\right] \cos \left[\frac{N}{C}\left(-i t-z_{0}+z\right)\right]}{\left[t^{2}+\left(z_{0}+z+\frac{C n \pi}{N}\right)^{2}\right] \cos \left[\frac{2 N}{C}\left(z+\frac{C n \pi}{N}\right)\right]}
$$

When $\lambda=\mu \sim O(\sqrt{N})$, the saddle point becomes $t_{0}=-i \lambda / \sqrt{N}$ and we set this value in $R$. The second pole gives a similar expression. Furthermore, if $C / N>>1$, we can approximate $\lambda \sim z_{0}+z+C n \pi / N$. Then, we find that the residue $R$ in (6.10) becomes $R=N / 2 C$. Therefore, we have the density of states for $C / N>>1$, and $\lambda \sim O(\sqrt{N})$, 


$$
\rho(\lambda)=\frac{\sqrt{N}}{4 C \sqrt{\pi}} \sum_{n}\left[e^{-\left(z_{0}+z+\frac{C n \pi}{N}-\lambda\right)^{2}}+e^{-\left(z_{0}-z+\frac{C(2 n+1) \pi}{2 N}-\lambda\right)^{2}}\right]
$$

which is a sum of Gaussian distributions.

In the clean case we have several impurities and one may average this density of states over $z$. As discussed in [11], the measure for this $z$-average is

$$
\rho_{0}(z)=A \cos ^{2}\left[\frac{2 N z}{C}\right]
$$

where $A=4 N / C \pi$. The matrix elements are represented by a quaternion, and the measure is isomorphic to the uniform measure over the four-dimensional sphere $s^{3}, S p(1) \sim S^{3}$. In spherical coordinate this leads to (6.13). When $C / N>>1$, the integration of $(6.12)$ over $z$ with the measure (6.14) yields

$$
\begin{aligned}
<\rho(z)> & =\int_{-\infty}^{\infty} e^{-\left(z_{0}+z+\frac{C n \pi}{N}-\lambda\right)^{2}} A \cos ^{2}\left(\frac{2 N z}{C}\right) d z \\
& =A \frac{\sqrt{\pi}}{2}\left[1-e^{-\frac{4 N^{2}}{C^{2}}} \cos \left(\frac{4 N}{C} \lambda\right)\right]
\end{aligned}
$$

(in which we have used $N / C<<1$ ). When $C / N \rightarrow \infty$, the exponential factor $\exp \left(-4 N^{2} / C^{2}\right)$ becomes one and the density of states becomes $\sin ^{2}(2 N \lambda / C)$. This is indeed the result for the clean case found in 11]. When $C / N$ is finite, at the energy $\lambda=n \pi C / 2 N=n \hbar \omega_{0}$, the density of state becomes non-vanishing, except at the origin. At the origin $\lambda=0$, we have a zero due to the factor $i t=\lambda$ in (6.11). It may be interesting to note this deviation from zero has been observed in the numerical work of [11].

When $C$ becomes order of $\sqrt{N}$, we may apply the saddle point method in (6.8). We take the saddle point equation (5.14) for $a_{\gamma}=\frac{\pi C}{2 N}\left(n-\frac{1}{2}+(-1)^{\gamma} \tilde{z}\right)$. However, the second and third term $\left(-1 / 2+(-1)^{\gamma} \tilde{z}\right)$ can be neglected since they remain of order one, compared to $n$ which can be order of $N$. Thus we may approximate $a-\gamma$ by $a_{\gamma}=\pi^{2} C^{2} n^{2} / 4 N^{3}$, which is independent of $z$. Therefore the average over $z$ does not change the result for the density of states, and we have a universal result for the kernel and for the density of states (5.13).

It is possible to take the average (6.10) by the formula,

$$
\int_{-\frac{\pi}{2}}^{\frac{\pi}{2}} d z \frac{a+\sin z}{b+\sin z} \cos ^{2} z=\pi\left[\frac{1}{2}+(a-b)\left(b-\sqrt{b^{2}-1}\right)\right]
$$

Then, changing the contour integration in the $v$-plane to the integration near the saddlepoints for $v$ in the large $\mathrm{N}$ limit, we have

$$
\begin{aligned}
K_{N}(\lambda, \mu) & =-\frac{C A \pi}{4 N^{2}} \int \frac{d t}{2 \pi} \int \frac{d v}{2 \pi}\left(\frac{t}{t^{2}+v^{2}}\right)\left[\left(\cosh \left(\frac{2 N t}{C}\right)-\cos \left(\frac{2 N v}{C}\right)\right)\right. \\
& \left.\times \sin \left(\frac{2 N v}{C}\right)\right] e^{-t^{2}-2 i t \mu-v^{2}+2 v \lambda}
\end{aligned}
$$

The term $\sin (2 N v / C)$ comes from the sum of integration paths in opposite directions. We assume here that $\lambda$ and $\mu$ are of order one, and $O(\lambda) \sim O\left(\frac{N}{C}\right)$. After the shift $t \rightarrow \sqrt{N} t$, the 
saddle-point is at $t_{0}=-i \mu / \sqrt{N} \pm \sqrt{N} / C$ and $v=\lambda / \sqrt{N} \pm i \sqrt{N} / C$ for the terms in (6.16). Inserting the values of these saddle-points, we have

$$
\begin{aligned}
\rho(\lambda) & =\frac{N}{C}\left[1-\frac{C}{4 N \lambda} \sin \left(\frac{4 N \lambda}{C}\right)\right]+\frac{C}{2 N} \sin ^{2}\left(\frac{2 N \lambda}{C}\right) \\
& -\frac{C \lambda^{2}}{4 N\left(\lambda^{2}+\frac{N^{2}}{C^{2}}\right)}\left[1+e^{-\frac{4 N^{2}}{C^{2}}} \cos \left(\frac{4 N \lambda}{C}\right)\right] \\
& +\frac{\lambda}{4\left(\lambda^{2}+\frac{N^{2}}{C^{2}}\right)} e^{-\frac{4 N^{2}}{C^{2}}} \sin \left(\frac{4 N \lambda}{C}\right)
\end{aligned}
$$

The above expression reduces to that of the dirty case when $C / N \rightarrow 0$, since the first term is then dominant. Thus (6.17) gives the correction to the dirty limit, and it is valid for $C \sim N$.

In the large $C, C>>N$, it is easy to recover the clean case from the expression (6.16). The saddle-point is given by $t=-i \mu$ and $v=\lambda$. Putting these values in (6.16), and taking $\mu \rightarrow \lambda$, we obtain immediately

$$
\rho(\lambda)=\frac{N}{2 C} \sin ^{2}\left(\frac{2 N \lambda}{C}\right)
$$

Thus, (6.16) gives the result both for the dirty case and for the clean case when one varies the parameter $C$.

\section{DISCUSSION}

We have discussed a random matrix theory for the energy levels inside a superconductor vortex and investigated the crossover from the clean case to the dirty case.

The technique involves a transposition of earlier results for the Hermitian case to the symplectic group $S p(N)$. An extension of the Itzykson-Zuber integral is presented (in an appendix). In the symplectic case we have to consider an external source matrix, which describes the spectrum of energies in the absence of impurities, whose eigenvalues are of the form $a=\left(a_{1}, \ldots, a_{N},-a_{1}, \ldots,-a_{N}\right)$. In the symplectic case the measure $\Delta(a)=\prod\left(a_{i}^{2}-a_{j}^{2}\right) \prod a_{i}$ replaces the Vandermonde determinant of the unitary case. Indeed, in the superconductor vortex, the eigenvalues appear in opposite pairs, i.e. with an exact particle-hole symmetry, as seen in the Andreev reflexion. We have then discussed generalized integral formulae related to the $S p(N)$ group. In an appendix we have investigated hypergeometric functions of matrix argument both for the unitary and symplectic groups. This result may be useful for non-Gaussian random distributions.

This random matrix theory is phenomenological. One phenomenological parameter, which can be viewed as the intensity of the disorder, is sufficient to describe the distribution functions of the random matrices. We have fixed this disorder parameter to one, which is why it does not appear explicitly in (4.2). Instead for the external source matrix $A$, we have introduced a parameter $C$ in (5.16). We find the a universal formula for the density of states and the correlation functions for the different regimes of this parameter: a clean case $C>N$, a dirty case $C<N$, and a crossover region $C \sim N$. Nevertherless the identification of this 
parameter $C$ with microscopic parameters, such as the strength of the impurity potential $V_{i}$ and the number of impurities $N_{i}$ in (2.4), requires a microscopic study which is outside the scope of this work.

\section{ACKNOWLEDGMENTS}

This work was supported by the CREST of JST. S. H. thanks a Grant-in-Aid for Scientific

Research by the Ministry of Education, Science and Culture. A. L. thanks a Grant NSF DMR-9812340. S. H. and A. L. thanks ICTP in Trieste where this work was started. 


\section{Appendix A: Harish-Chandra formula for the unitary, orthogonal and sym-} plectic group

Let $G$ be a compact connected Lie group. The Harish-Chandra integral formula is [6.17, 18]

$$
\int_{G} e^{<\operatorname{Ad}(g) \cdot a|b\rangle} d g=\frac{\sum_{w \in W}(\operatorname{det} w) e^{<w \cdot a \mid b>}}{\Delta(a) \Delta(b)}
$$

where $a, b \in h ; h$ is a Lie algebra, and for any $H \in h$,

$$
\Delta(H)=\prod_{\alpha \in \Delta_{+}} \alpha(H)
$$

$\Delta_{+}$is the collection of positive roots, $W$ is the finite reflection group, called the Weyl (or Coxeter) group and $h$ is the Cartan subalgebra of the Lie algebra of the group. In the general classification theory, the irreducible finite refelection groups are categorized as belonging to various types, $A_{n}, B_{n}, C_{n}, D_{n}, \ldots$, which are associated with certain compact Lie groups. In (3.4), $\operatorname{det}(w)$ is simply \pm 1 , since each $w \in W$ is an orthogonal transformation.

Let us illustrate this result in the simplest case of the unitary group. The Lie algebra of $S U(n), u=s u(n)$, consists $n \times n$ complex skew-Hermitian traceless matrices. The complexification of $u$ is $g=\operatorname{sl}(n, C)$, the Lie algebra of all $n \times n$ complex matrices with zero trace. The Cartan subalgebra $h$ of $g$ consists of diagonal $n \times n$ complex matrices $H=\operatorname{diag}\left(h_{1}, \ldots, h_{n}\right)$ such that $h_{1}+\ldots .+h_{n}=0$. We define the linear functional $e_{j}$ on $h$ by $e_{j}(H)=h_{j}$, and the $n \times n$ matrix $E_{j k}$ which consists of 1 in the $(\mathrm{j}, \mathrm{k})$ th position and 0 elsewhere. The linear functional $\alpha=e_{j}-e_{k}, j \neq k$ is a root of $g$ with respect to $h$, i.e. $\Delta=\left[e_{j}-e_{k}: 1 \leq j \neq k \leq n\right]$. One then verifies that (A.1) reduces the Itzykson-Zuber formula (3.4) in the unitary case.

The orthogonal group $O(N)$ consists of real $N \times N$ matrices $u$ such that $u u^{t}=1$. The Harish-Chandra formula applies to compact connected groups $G$ and we thus restrict ourselves to the special orthogonal subgroup $S O(N)$, of orthogonal matrices with determinant one. The Lie algebra $g=s o(N)$ of $G=S O(N)$ consists of all $N \times N$ real skew-symmetric traceless matrices. We need to consider separately the even case $N=2 n$ and the odd one $N=2 n+1$.

For $S O(2 n)$, the Cartan subalgebra $h$ is the complex Lie algebra of all $2 n \times 2 n$ complex block-diagonal matrices of the form [22]

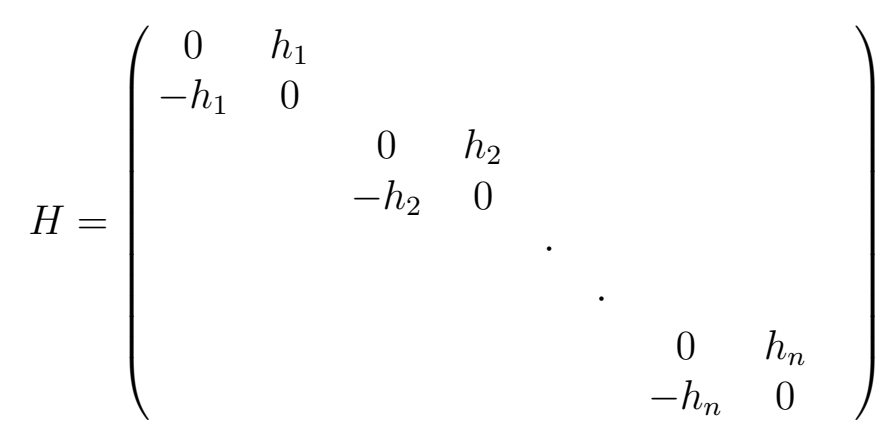

This matrix $H$ is written as the direct sum of $v$ 's defined as 


$$
\begin{gathered}
v=\left(\begin{array}{cc}
0 & 1 \\
-1 & 0
\end{array}\right) \\
H=h_{1} v \oplus h_{2} v \oplus \cdots \oplus h_{n} v
\end{gathered}
$$

Let $\left[e_{1}, \ldots, e_{n}\right]$ be the standard basis for $R^{n}$. A root system for $S O(2 n)$ is $\Delta=\left[ \pm e_{j} \pm e_{k}\right.$ : $1 \leq j \leq k \leq n]$, which is a root system of type $D_{n}$. For $\alpha=e_{j} \pm e_{k} \in \Delta_{+}$and the Cartan subalgebra $H$, we have $\alpha(H)=h_{j} \pm h_{k}$ and

$$
V(H)=\prod_{1 \leq j<k \leq n}\left(h_{j}^{2}-h_{k}^{2}\right) .
$$

A fundamental Weyl chamber is defined as $S=\left[\left(h_{1}, \ldots, h_{n}\right) \in R^{n}: h_{1}>\cdots>h_{n-1}>\left|h_{n}\right|\right]$. The group $G(n)$ of permutations $w$ of the set $[-n, \ldots,-1,1, \ldots, n]$ restricted to $w(-j)=-$ $w(j)$, acts on the set of $\left[h_{-n}, \ldots, h_{-1}, h_{1}, \ldots, h_{n}\right]$ as

$$
w \cdot\left(h_{1}, \ldots, h_{n}\right)=\left(h_{w(1)}, \ldots, h_{w(n)}\right)
$$

where we denote $h_{-j}=-h_{j}, j=1, \ldots, n$. The Weyl group $W$ consists of $W=$ [permutations and even number of sign changes of $\left.\left[e_{1}, \ldots, e_{n}\right]\right]$; thus $|W|=2^{n-1} n$ !. For $H$, we have

$$
w \cdot H=h_{w(1)} v \oplus h_{w(2)} v \oplus \cdots \oplus h_{w(n)} v
$$

Therefore the Harish-Chandra formula (A.1) gives for $a=a_{1} v \oplus a_{2} v \oplus \cdots \oplus a_{n} v$ and $b=$ $b_{1} v \oplus b_{2} v \oplus \cdots \oplus b_{n} v$

$$
\int_{S O(2 n)} e^{\operatorname{tr}\left(g a g^{-1} b\right)} d g=C_{S G(n)} \frac{\sum_{w \in S G(n)}(\operatorname{det} w) \exp \left(2 \sum_{j=1}^{n} w\left(a_{j}\right) b_{j}\right)}{\prod_{1 \leq j<k \leq n}\left(a_{j}^{2}-a_{k}^{2}\right)\left(b_{j}^{2}-b_{k}^{2}\right)}
$$

with $C_{S G(n)}=(2 n-1) ! \prod_{j=1}^{2 n-1}(2 j-1)$ !.

For $S O(2 n+1)$, the matrix $H$ is

$$
H=h_{1} v \oplus h_{2} v \oplus \cdots \oplus h_{n} v \oplus 0
$$

and the root system for $S O(2 n+1)$ is

$$
\Delta=\left[ \pm e_{j} \pm e_{k}: 1 \leq j \leq k \leq n\right] \cup\left[ \pm e_{j}: 1 \leq j \leq n\right]
$$

Thus for $\alpha \in \Delta_{+}$and $H$, we have

$$
\begin{array}{cc}
\alpha(H)=h_{j} \pm h_{k} & \text { if } \quad \alpha=e_{j} \pm e_{k} \\
h_{j} & \text { if } \quad \alpha=e_{j}
\end{array}
$$

and

$$
V(H)=\prod_{1 \leq j<k \leq n}\left(h_{j}^{2}-h_{k}^{2}\right) \prod_{j=1}^{n} h_{j}
$$


The weyl group of $S O(2 n+1)$ is $W=$ [ permutations and sign changes of $\left.\left[e_{1}, \ldots, e_{n}\right]\right] .|W|=$ $2^{n} n$ !.

The action of $G(n)$ on the Lie algebra $h$ is

$$
w \cdot H=h_{w(1)} v \oplus h_{w(2)} v \oplus \cdots \oplus h_{w(n)} v \oplus 0
$$

The Harish-Chandra formula (3.4) becomes for $a=a_{1} v \oplus \cdots \oplus a_{n} v \oplus 0, b=b_{1} v \oplus \cdots \oplus b_{n} v \oplus 0$,

$$
\int_{S O(2 n+1)} e^{\operatorname{tr}\left(g a g^{-1} b\right)} d g=C_{G(n)} \frac{\sum_{w \in G(n)}(\operatorname{det} w) \exp \left(2 \sum_{j=1}^{n} w\left(a_{j}\right) b_{j}\right)}{\prod_{1 \leq j \leq k \leq n}\left(a_{j}^{2}-a_{k}^{2}\right)\left(b_{j}^{2}-b_{k}^{2}\right) \prod_{j=1}^{n} a_{j} b_{j}}
$$

where $C_{G(n)}=\prod_{j=1}^{n}(2 j-1) ! \prod_{j=2 n}^{4 n-1} j !$.

For the case of the symplectic group $S p(N)$, we have

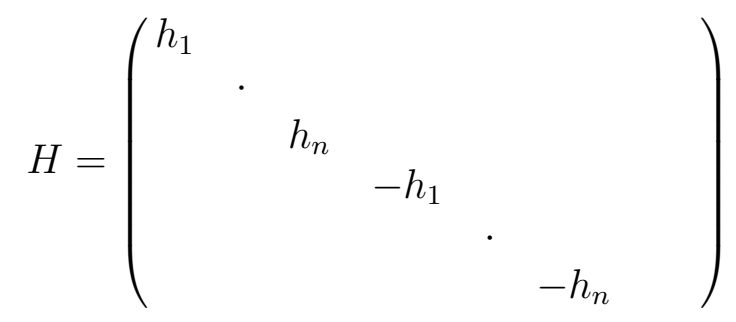

and $e_{j}(H)=h_{j}$. A root system for $S p(N)$ is

$$
\Delta=\left[ \pm e_{j} \pm e_{k}: 1 \leq j \leq k \leq n\right] \cup\left[ \pm 2 e_{j}: 1 \leq j \leq n\right]
$$

The Weyl group for the $\operatorname{Sp}(\mathrm{N})$ algebra is $W=$ [permutations and sign changes of $\left.\left[e_{1}, \ldots, e_{n}\right]\right]$. $|W|=2^{n} n !$.

\section{Appendix B: Generalization of the Itzykson-Zuber formula}

The Itzykson-Zuber formula for Hermitian matrices $a$ and $b$ is again

$$
\int_{U(n)} e^{\operatorname{tr}\left(g a g^{-1} b\right)} d g=\frac{\operatorname{det}\left(e^{a_{i} b_{j}}\right)}{\Delta(a) \Delta(b)}
$$

where $a_{i}$ and $b_{i}$ are eigenvalues of $a$ and $b$, respectively. We wish now to consider generalizations of this type of integrals for which

$$
\int_{U(n)} \psi_{n}\left(g a g^{-1} b\right) d g=\frac{\operatorname{det}\left(f\left(a_{i} b_{j}\right)\right)}{\Delta(a) \Delta(b)}
$$

where $\psi_{n}$ is a function of a matrix argument, and $f$ is a real function.

The Itzykson-Zuber formula of (B.1) corresponds to $f(x y)=\exp [x y]$ and $\psi_{n}=$ $\exp \left[\operatorname{tr}\left(g a g^{-1} b\right)\right]$.

We may take for $f(x y)$ an hypergeometric function ${ }_{p} \mathcal{F}_{q}\left(\alpha_{1}, \ldots, \alpha_{p} ; \beta_{1}, \ldots, \beta_{q} ; x y\right)$. Note that $f(x y)=\exp [x y]={ }_{0} \mathcal{F}_{0}(x y)$. Then , the corresponding function $\psi_{n}(t)$ is also an hypergeometric function of the matrix argument ${ }_{p} F_{q}\left(\alpha_{1}+n-1, \ldots, \alpha_{p}+n-1 ; \beta_{1}+n-1, \ldots, \beta_{q}+n-1 ; t\right)$. For example,in the $p=1, q=0$ case, we have 


$$
\begin{aligned}
\int_{U(n)} \operatorname{det}\left(1-a g b g^{-1}\right)^{-\alpha-n+1} d g & ={ }_{1} F_{0}(\alpha ; a, b) \\
& =\frac{\operatorname{det}\left({ }_{1} \mathcal{F}_{0}\left(\alpha ; a_{i} b_{j}\right)\right)}{\Delta(a) \Delta(b)}
\end{aligned}
$$

where ${ }_{1} \mathcal{F}_{0}\left(\alpha ; a_{i} b_{j}\right)=\left(1-a_{i} b_{j}\right)^{-\alpha}$. This is easily checked directly for the $n=2, g=U(2)$ case. We represent $g$ as

$$
g=\left(\begin{array}{cc}
\cos \phi e^{i \theta_{1}} & \sin \phi e^{i \theta_{2}} \\
-\sin \phi e^{i \theta_{3}} & \cos \phi e^{-i\left(\theta_{1}-\theta_{2}-\theta_{3}\right)}
\end{array}\right)
$$

with the measure $J=\cos \phi \sin \phi d \phi \prod_{i=1}^{3} d \theta_{i}$.

More generally, the formula of the integration over the $U(N)$ group may be written as

$$
\begin{aligned}
& { }_{p} F_{q}\left(\alpha_{1}, \ldots, \alpha_{p} ; \beta_{1}, \ldots, \beta_{q} ; a, b\right) \\
= & \int_{U(n)}{ }_{p} F_{q}\left(\alpha_{1}, \ldots, \alpha_{p} ; \beta_{1}, \ldots, \beta_{q} ; a g b g^{-1}\right) d g \\
= & C \frac{\operatorname{det}\left({ }_{p} \mathcal{F}_{q}\left(\alpha_{1}-n+1, \ldots, \alpha_{p}-n+1 ; \beta_{1}-n+1, \ldots, \beta_{q}-n+1 ; a_{i} b_{j}\right)\right)}{\Delta(a) \Delta(b)}
\end{aligned}
$$

This derivation of (B.5) proceeds by induction [25]. First, we introduce the zonal polynomials $Z_{m}(A)$ [24] which are homogeneous polynomials of degree $\mathrm{m}$, which are symmetric functions of the $n$ eigenvalues of the matrix $A$. From their definition they have the simple property that

$$
\int_{G} Z_{m}\left(g A g^{-1} B\right) d g=\frac{Z_{m}(A) Z_{m}(B)}{Z_{m}(1)}
$$

in which the integral runs over the elements of a compact Lie group $G$ with a Haar measure normalized to one. The coefficients of these polynomials are group-dependent, but they can be constructed from this property inductively. The polynomials are thus expressed as decompositions of products of $\operatorname{Tr}\left(A_{1}^{m}\right) \operatorname{Tr}\left(A_{2}^{m}\right) \cdots \operatorname{Tr}\left(A_{n}^{m}\right)$, with $|m|=m_{1}+\cdots+m_{n}$, and the $m_{j}$ are the partitions of $\mathrm{m}$ characterizing a Young tableau. It then follows that

$$
(\operatorname{tr} A)^{k}=\sum_{|m|=k} Z_{m}(A)
$$

in which the sum runs over all the Young tableaux with $\mathrm{k}$ boxes. We may now take for a generating function the hypergeometric function with matrix argument defined as

$$
{ }_{p} F_{q}\left(\alpha_{1}, \ldots, \alpha_{p} ; \beta_{1}, \ldots, \beta_{q} ; t\right)=\sum_{k=0}^{\infty} \frac{1}{k !} \sum_{|m|=k} \frac{\left[\alpha_{1}\right]_{m} \cdots\left[\alpha_{p}\right]_{m}}{\left[\beta_{1}\right]_{m} \cdots\left[\beta_{q}\right]_{m}} Z_{m}(t) .
$$

Then, one has

$$
\begin{aligned}
{ }_{p} F_{q}\left(\alpha_{1}, \ldots, \alpha_{p} ; \beta_{1}, \ldots, \beta_{q} ; a, b\right) & =\int_{G}{ }_{p} F_{q}\left(\alpha_{1}, \ldots, \alpha_{p} ; \beta_{1}, \ldots, \beta_{q} ; g a g^{-1} b\right) d g \\
& =\sum_{k=0}^{\infty} \frac{1}{k !} \sum_{|m|=k} \frac{\left[\alpha_{1}\right]_{m} \cdots\left[\alpha_{p}\right]_{m}}{\left[\beta_{1}\right]_{m} \cdots\left[\beta_{q}\right]_{m}} \frac{(a) Z_{m}(b)}{Z_{m}(1)}
\end{aligned}
$$


where

$$
\begin{gathered}
{[\alpha]_{m}=\prod_{j=1}^{n}(\alpha-j+1)_{m_{j}}} \\
(\alpha)_{k}=\alpha(\alpha+1) \cdots(\alpha+k-1)
\end{gathered}
$$

In the case of the unitary group $G=U(n)$ the zonal polynomial $Z_{m}(A)$ is a Schur function, namely it is given by the Weyl formula for the characters of the representations of $G$

$$
Z_{m}(A)=\frac{\operatorname{det}\left(a_{i}^{m_{j}+n-j}\right)}{\operatorname{det}\left(a_{i}^{n-j}\right)} .
$$

The Euler integral gives

$$
Z_{m}(A)=\frac{\Gamma_{n}(\beta)}{\Gamma_{n}(\beta-\alpha) \Gamma_{n}(\alpha)} \frac{[\beta]_{m}}{[\alpha]_{m}} \int_{0<r<1} Z_{m}(r A) \operatorname{det}(r)^{\alpha-n} \operatorname{det}(1-r)^{\beta-\alpha-n} d r
$$

where the integration is over Hermitian matrices $r$ whose eigenvalues are between 0 and 1 . The Gamma function $\Gamma_{n}(\alpha)$ is defined by

$$
\Gamma_{n}(\alpha)=\pi^{n(n-1) / 2} \prod_{i=1}^{n} \Gamma(\alpha-i+1)
$$

Using these notations, we have established the recurrence formula,

$$
\begin{aligned}
& { }_{p+1} F_{q+1}\left(\alpha_{1}, \ldots, \alpha_{p+1} ; \beta_{1}, \ldots, \beta_{q+1} ; a, b\right)=\frac{\Gamma_{n}\left(\beta_{q+1}\right)}{\Gamma_{n}\left(\alpha_{p+1}\right) \Gamma_{n}\left(\beta_{q+1}-\alpha_{p+1}\right)} \\
& \times \int_{0<r<1} \operatorname{det}(r)^{\alpha_{p+1}-n} \operatorname{det}(1-r)^{\beta_{q+1}-\alpha_{p+1}-n}{ }_{p} F_{q}\left(\alpha_{1}, . ., \alpha_{p} ; \beta_{1}, . ., \beta_{q} ; r a, b\right) d r
\end{aligned}
$$

which proves (B.5) inductively.

For the case of the confluent and Gaussian hypergeometric functions, ${ }_{1} F_{1}$ and ${ }_{2} F_{1}$, these formulae reduce to

$$
\begin{aligned}
& { }_{1} F_{1}(\alpha ; \beta ; t)=\frac{\Gamma_{n}(\beta)}{\Gamma_{n}(\alpha) \Gamma_{n}(\beta-\alpha)} \int_{0<r<1} d r e^{\operatorname{tr}(r t)} \operatorname{det}(r)^{\alpha-n} \operatorname{det}(1-r)^{\beta-\alpha-n} \\
& { }_{2} F_{1}(\alpha, \beta ; \gamma ; t)=\frac{\Gamma_{n}(\gamma)}{\Gamma_{n}(\beta) \Gamma_{n}(\gamma-\beta)} \int_{0<r<1} d r \operatorname{det}(r)^{\beta-n} \operatorname{det}(1-r)^{\gamma-\beta-n} \\
& \times \operatorname{det}(1-r t)^{-\alpha}
\end{aligned}
$$

For the symplectic group $S p(n)$, we have similar formulae with hypergeometric functions. In the case $p=0, q=0$, and $p=1, q=0$, which is similar to (B.1) and (B.3), 


$$
\begin{aligned}
{ }_{0} F_{0}(a, b) & =\int_{S p(n)} e^{\operatorname{tr}\left(g a g^{-1} b\right)} d g \\
& =\frac{\operatorname{det}\left[2 \sinh \left(2 a_{i} b_{j}\right)\right]}{\Delta(a) \Delta(b)} \\
{ }_{1} F_{0}(\alpha ; a, b) & =\int_{S p(n)} \operatorname{det}\left(1-a g b g^{-1}\right)^{-\alpha-2 n+1} \mathrm{~d} g \\
& =\frac{\operatorname{det}\left[\left(\frac{1}{1-a_{i} b_{j}}\right)^{2 \alpha}-\left(\frac{1}{1+a_{i} b_{j}}\right)^{2 \alpha}\right]}{\Delta(a) \Delta(b)}
\end{aligned}
$$

where $\Delta(a)=\prod\left(a_{i}^{2}-a_{j}^{2}\right) \prod a_{i}$. These formula are easily checked for the simplest case, $n=1$, $S p(1)$, which is isomorphic to $S U(2)$, with

$$
g=\left(\begin{array}{cc}
\cos \phi e^{i \theta_{1}} & \sin \phi e^{i \theta_{2}} \\
-\sin \phi e^{-i \theta_{2}} & \cos \phi e^{-i \theta_{1}}
\end{array}\right)
$$

It may be useful to notice that these results for $S p(1)$ may also be derived immediately from the $U(2)$ case by setting $a=a_{1}=-a_{2}$, and $b=b_{1}=-b_{2}$ in (B.1) and (B.3). Indeed the Lie group $g$ of $S p(1)$ in $(\overline{B .20})$ is derived from (B.4) with the condition $\theta_{3}=-\theta_{2}$. The denominator $\Delta(a)$ for $S p(n)$ is also derived from the $U(n)$ case with the condition on the eigenvalues $a=\operatorname{diag}\left(a_{1}, \cdots, a_{n},-a_{1}, \cdots,-a_{n}\right)$. This equivalence between $U(n)$ and $S p(n)$ holds for $n \neq 1$. Since the eigenvalues appear here in pairs $\left(a_{i},-a_{i}\right)$, we have to replace $n$ by $2 n$ in the hypergeometric relations as shown in (B.19). For a general hypergeometric function, we have for the $S p(n)$ case,

$$
\begin{aligned}
& { }_{p} F_{q}\left(\alpha_{1}, \ldots, \alpha_{p} ; \beta_{1}, \ldots, \beta_{q} ; a, b\right) \\
= & \int_{S p(n)}{ }_{p} F_{q}\left(\alpha_{1}, \ldots, \alpha_{p} ; \beta_{1}, \ldots, \beta_{q} ; a g b g^{-1}\right) d g \\
= & \frac{C}{\Delta(a) \Delta(b)} \operatorname{det}\left[\left({ } _ { p } \mathcal { F } _ { q } \left(\alpha_{1}-2 n+1, \ldots, \alpha_{p}-2 n+1 ; \beta_{1}-2 n+1, \ldots,\right.\right.\right. \\
& \left.\left.\beta_{q}-2 n+1 ; a_{i} b_{j}\right)\right)^{2} \\
- & \left({ } _ { p } \mathcal { F } _ { q } \left(\alpha_{1}-2 n+1, \ldots, \alpha_{p}-2 n+1 ; \beta_{1}-2 n+1, \ldots,\right.\right. \\
& \left.\left.\left.\beta_{q}-2 n+1 ;-a_{i} b_{j}\right)\right)^{2}\right]
\end{aligned}
$$




\section{REFERENCES}

[1] C. Caroli, P. G. De Gennes and J. Matricon, Phys. Lett. 9, (1964) 307.

[2] E. Brézin and S. Hikami, Nucl. Phys. B479 (1996) 697.

[3] E. Brézin, S. Hikami and A. Zee, Nucl. Phys. 464 (1996) 411.

[4] E. Brézin and S. Hikami, Phys. Rev. E 55 (1997) 4067.

[5] E. Brézin and S. Hikami, Phys. Rev. E 56264 (1997).

[6] Harish-Chandra, Proc. Nat. Acad. Sci. 42252 (1956).

[7] C. Itzykson and J. -B. Zuber, J. Math. Phys. 21 (1980) 411.

[8] J. J. Duistermaat and G. H. Heckman, Invent. Math. 69 (1982) 259.

[9] V. A. Kazakov, Nucl. Phys. B 354 (1991) 697.

[10] M.L. Mehta, Random Matrices, Academic Press (1991)

[11] A. A. Koulakov and A. I. Larkin, cond-mat/9810125.

[12] A. I. Larkin and Yu. N. Ovchinnikov, Phys. Rev. B 57 (1998) 5457

[13] M. A. Skvortsov, V. E. Kravtsov and M. V. Feigel'man, cond-mat/9805296.

[14] A. A. Koulakov and A. I. Larkin, cond-mat/9802002.

[15] A. Altland and M. R. Zirnbauer, Phys. Rev. Lett. 76 (1996) 3420.

[16] A. Altland, B. D. Simon and D. Taras-Semchuk, cond-mat/9807371.

[17] D. Altshuler and C. Itzykson, Ann. Inst. Heri Poincaré, 54 (1991) 1.

[18] K. I. Gross and D. S. P. Richards, J. Approx. Theory 59 (1989) 224, J. Approx. Theory 82(1995) 60.

[19] E. Witten, J. Geom. Phys. 9 (1992) 303.

[20] M. F. Atiyah and R. Bott, Topology 23 (1984) 1.

[21] S. Hikami and T. Maskawa, Prog. Theor. Phys. 67 (1982) 1038.

[22] A. W. Knapp, Representation theory of semisimple groups, Princeton University Press, Princeton, New Jersey 1986.

[23] Erdelyi et al. Higher transcendental functions, Vol.2, P.199, (1953) McGraw-Hill, New York.

[24] A. T. James, Ann. Math. Statist. 35 (1964) 475.

[25] K. I. Gross and D. S. P. Richards, Trans. American Math. Soc. 301 (1987) 781. 